\title{
Staphylococcus aureus - A Versatile Pathogen Biochemical Characterization and Antibiogram
}

\author{
A.W. Samira ${ }^{1}$, Samith Ahmed ${ }^{2 *}$ and Meera D. Meundi ${ }^{1}$ \\ ${ }^{1}$ Department of Microbiology, All Saints University School of Medicine, \\ Hillsborough Street, Roseau, Dominica, West Indies \\ ${ }^{2}$ Department of Pathology, All Saints University School of Medicine, \\ Hillsborough Street, Roseau, Dominica, West Indies \\ *Corresponding author
}

\section{Keywords}

S. aureus,

Coagulase test, MRSA

\section{Article Info}

Accepted:

26 January 2018

Available Online:

10 February 2018

\section{A B S T R A C T}

S. aureus is a major human pathogen capable of causing a wide range of infections and remains the second cause of nosocomial infections. To biochemically characterise the isolated coagulase-positive staphylococci, to determine its antimicrobial susceptibility and the MRSA status. 2220 samples were processed in the study period among which 150 were $S$. aureus, identified by Gram staining and coagulase test. Tests for characterisation included mannitol fermentation, demonstration of pigment production on milk agar, phosphatase test, DNase test, tellurite reduction test and urease production. Anti-microbial susceptibility was done using the commonly used antibiotics and MRSA (Methicillinresistant Staphylococcus aureus) status was determined by cefoxitin disc diffusion method. Of the 150 tube coagulase positive $S$. aureus, only 145 (96.7\%) were haemolytic, which were slide coagulase positive too in the first minute. The remaining 5 were slide coagulase positive after 1 minute. The positivity of $150 \mathrm{~S}$. aureus to the other pathogenicity tests were mannitol fermentation $(100 \%)$, DNase test $(100 \%)$, pigment production on milk agar (100\%), tellurite reduction (95.3\%), phosphatase production $(92 \%)$ and urease test (90.7\%). Out of the 150 S. aureus, $54 \%$ were MRSA. All the S. aureus isolates were resistant to ampicillin and cephalexin, partially resistant to other antibiotics and completely sensitive to vancomycin and linezolid. The MRSA isolates were resistant to cotrimoxazole, chloramphenicol, ciprofloxacin, tetracycline, gentamicin, erythromycin and clindamycin. The methicillin-sensitive strains were resistant to the above mentioned antibiotics but with a lower percentage; difference being about half the total percentage.

\section{Introduction}

Staphylococcus aureus (S. aureus) is normally a ubiquitous, but relatively innocent commensal and coloniser of the skin and mucosa of humans and several animal species which is in apparent contrast to its infectious potential (Van Belkum et al., 2009). S. aureus has been demonstrated to be a major human pathogen capable of causing a wide range of infections, from relatively mild skin infections such as folliculitis and furunculosis to lifethreatening conditions, including sepsis, deep abscesses, pneumonia, osteomyelitis, blood 
stream infections and infective endocarditis through both toxin-mediated and non-toxinmediated mechanisms (Van Belkum et al., 2009; Moreillon et al., 2005; Lowy, 2012).

S. aureus, has demonstrated its versatility by remaining a major cause of morbidity and mortality despite the availability of numerous effective anti-staphylococcal drugs (Lowy, 2012).

The first case of methicillin-resistant $S$. aureus (MRSA) was described in the United Kingdom in 1961. Currently, nosocomial MRSA rates approach $60 \%$ or more in many areas of the country (National nosocomial infections surveillance report, 2004).

The emergence of resistant strains represents a consequential response to selective pressures imposed by antimicrobial chemotherapy and once established, they are difficult to control and eradicate (Saikia et al., 2009).

Most isolates remain susceptible to glycopeptides (vancomycin, teicoplanin), oxazolidinones (linezolid), streptograminins (quinupristin-dalfopristin), and polycyclic compounds (tetracycline, tigecycline) (Moreillon et al., 2005; Deresinski, 2005). Low level resistance even to vancomycin is emerging at present (Assadullah et al., 2003). The prevalence of MRSA strains is reported to be increasing.

Interestingly, there appears to be significant variable in the epidemiology and prevalence of MRSA in different parts of the world and even in different regions of a country (Voss $e t$ al., 1994). Detection of mecA gene or its product, penicillin binding proteins (PBP 2a), is considered the gold standard for MRSA confirmation (Skov et al., 2006). Results of cefoxitin disc diffusion test is in concordance with the PCR for mecA gene, and thus the cefoxitin disc diffusion method is very suitable for detection of MRSA and the test can be an alternative to PCR for detection of MRSA in resource constraint settings (Anand et al., 2009). Constant monitoring of these strains is essential in order to control their spread in hospital environment and transmission to community (Naseer and Jayaraj, 2010). Hence, the present study is undertaken to know the cultural characteristics, and to study the antibiotic sensitivity pattern of $S$. aureus isolated from clinical samples in our hospital with special reference to methicillin-resistant $S$. aureus.

\section{Materials and Methods}

This study was conducted for a period of one and half year; from November 2011 to May 2013 in the Department of Microbiology, K.V.G Medical College and Hospital. $S$. aureus strains isolated from all clinical specimens received during the study period to microbiology laboratory were analysed.

\section{Specimen collection}

Pus, discharge from skin and soft tissue infections, swabs from ears, conjunctiva and umbilicus, urine and blood obtained from patients of K.V.G Medical College Hospital were included in the study Identification of $S$. aureus was done by standard procedure. Tests for characterisation included catalase test, coagulase tests- slide and tube, mannitol fermentation, demonstration of pigment production on milk agar, phosphatase test, DNase test, tellurite reduction test and urease production.

Antimicrobial susceptibility tests were carried out by Kirby-Bauer disc diffusion method on Mueller-Hinton agar. The following discs (HiMedia, Mumbai) were used: Ampicillin $(10 \mu \mathrm{g})$, Cephalexin $(30 \mu \mathrm{g})$, Cotrimoxazole $(25 \mu \mathrm{g}), \quad$ Chloramphenicol $\quad(30 \mu \mathrm{g})$, Ciprofloxacin $(5 \mu \mathrm{g})$, Pristinamycin $(15 \mu \mathrm{g})$, 
Linezolid $\quad(30 \mu \mathrm{g}), \quad$ Amikacin $\quad(30 \mu \mathrm{g})$,

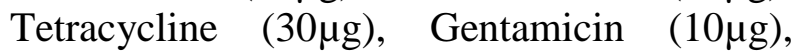
Erythromycin $(15 \mu \mathrm{g})$, Clindamycin $(2 \mu \mathrm{g})$, and Vancomycin $(30 \mu \mathrm{g})$.

Methicillin resistance was screened by disc diffusion method using $30 \mu \mathrm{g}$ cefoxitin disc. The diameter of the zone of inhibition was measured and interpretation was done in accordance with the CLSI guidelines. An isolate was considered to be a MRSA strain if cefoxitin inhibition zone diameter was $<21$ $\mathrm{mm}$.

\section{Analysis of data}

The data was analysed on IBM SPSS version 19. Chi-square test was applied to test the difference between proportions, at 5\% level of significance.

\section{Results and Discussion}

A total of 2220 samples were processed in the Department of Microbiology during the study period, which included 965 urine samples, 531 pus and wound swabs, 372 blood samples, 287 sputum and 65 body fluids (Fig. 1).

Of the 150 tube coagulase positive $S$. aureus, only $145(96.7 \%)$ were haemolytic, which were slide coagulase positive too in the first minute. The remaining 5 were slide coagulase positive after 1 minute.

The positivity of $150 \mathrm{~S}$. aureus of other pathogenicity tests were mannitol fermentation (100\%), DNase test (100\%), pigment production on milk agar (100\%), tellurite reduction (95.3\%), phosphatase production (92.\%) and urease test (90.7\%).

Of the 150 strains of S. aureus, 97 (65\%) were from male patients, 53 (35\%) were from female patients. Majority of the isolates of $S$. aureus were from pus $(87.3 \%)$. The infection of other anatomical sites all put together was only $12.7 \%$.

Among the battery of antibiotics used, $S$. aureus exhibited complete susceptibility to vancomycin (100\%) and linezolid (100\%). Next in the susceptibility order were pristinamycin (84.7\%), amikacin (66.7\%), clindamycin $(56.7 \%)$, tetracycline $(56.7 \%)$ and cotrimoxazole $(50.7 \%)$. Less than $50 \%$ susceptibility was observed with erythromycin (38\%), chloramphenicol (38\%), ciprofloxacin $(35.3 \%)$ and gentamicin (22.7\%). A 100\% resistance was shown to ampicillin and $1^{\text {st }}$ generation antibiotic, namely cephalexin. All the $S$. aureus were subjected to cefoxitin disc diffusion for detection of methicillin resistance (Fig. 2).

Sixty nine (46\%) strains of the 150 isolates of $S$. aureus were methicillin-resistant (MRSA) by cefoxitin disc diffusion method

S. aureus isolated from pus was $88 \%, 50 \%$ being MRSA and $38 \%$ being MSSA (Fig. 3).

MRSA infections of the in-patients were highest after the 4th day (25.2\%) of hospitalisation, which was highly significant (p value 0.001). In contrast, MSSA were highest on day $1(26.2 \%)$ and by 4 th day reduced to zero (Fig. 4).

Figure 5 illustrates the pattern of susceptibility of MRSA and MSSA isolates to other antibiotics. While both MRSA and MSSA were $100 \%$ sensitive to vancomycin and linezolid, $83.9 \%$ and $85.6 \%$ respectively were sensitive to pristinamycin. Next in the susceptibility order were amikacin $(69.2 \%$ and $63.7 \%$ ), clindamycin $(51.8 \%$ and $62.3 \%$ ) for MRSA and MSSA respectively. Less than $50 \%$ susceptibility was observed with chloramphenicol $(38.3 \%$ and $37.7 \%)$, cotrimoxazole $(29.6 \%$ and $49.3 \%)$, erythromycin $(28.4 \%$ and $49.3 \%)$, and 
ciprofloxacin (23.4\% and 49.3\%) for MSSA and MRSA respectively. A very high sensitivity pattern to tetracycline was observed for MSSA (92.8\%) as compared to MRSA (25.9\%). Both MSSA and MRSA were totally resistant to ampicillin and cephalexin.

Out of the 150 patients from whom S. aureus was isolated, 43 had the history of antibiotic use earlier (Fig. 6).

All the $43 \mathrm{~S}$. aureus isolated from these patients were MRSA, which is highly significant ( $\mathrm{p}$ value 0.001 ).

$S$. aureus is the most important nosocomial pathogen because of both the diversity and the severity of the infections it causes, including superficial, deep skin, and soft-tissue infections, endocarditis, and bacteremia, as well as a variety of toxin-mediated diseases such as gastroenteritis, staphylococcal scalded-skin syndrome, and toxic shock syndrome.

Coagulase is an established pathogenicity test, done on spot for all staphylococci. Initially, the slide coagulase test is done which is followed by the tube coagulase for confirmation. In our study, all the $S$. aureus gave positive result for the coagulase test by both the methods. Earlier reports (Rajaduraipandi et al., 2006; Brown and Ngeno; Shanthi and Sekar, 2009; Arora et al., 2010; Rahbar and Hajia, 2007; Han et al., 2007) too have established the high quality of coagulase test in establishing pathogenicity of $S$. aureus. Hence, the method is considered a 'gold standard' in the identification of $S$. aureus.

Mannitol fermentation, DNase and coagulase tests are the pathogenicity tests of importance for $S$. aureus. We observed that, both DNase production and mannitol fermentation were as sensitive as coagulase test in establishing the pathogenicity of S. aureus. (Han et al., 2007) reported only $99.6 \%$ positivity for $S$. aureus for mannitol fermentation test. Other pathogenicity tests for $S$. aureus such as production of pigment on milk agar (100\%), phosphatase enzyme (92\%) and reduction of tellurite to tellurium (95.3\%), also showed high positivity though not $100 \%$.

All the $S$. aureus have shown pigment production on milk agar but there was a slight variation in the tinge of golden-yellow colour. $86.7 \%$ of $S$. aureus demonstrated the production of golden-yellow pigment, the rest of the $13.3 \%$ produced a light-yellow pigment - which is definitely a feature of pathogenicity.

As compared to all the other characteristics, urease test shows the variation- $90 \%$ were positive, $10 \%$ were negative. Similar findings were observed by Udo et al., (2006), where urease positivity was $62 \%$.

There is a significant difference between $S$. aureus amongst males (65\%) and females (35\%), which is almost similar to Tsering et al., (2011).

Our isolation rate of $S$. aureus from pus is also on the higher side of the spectrum (87\%). We have isolated S. aureus from $4.7 \%$ of urine samples which is comparable to the studies done by Anupurba et al., (2003), Shanthi and Sekar (2009) and Velvizhi et al., (2011). S. aureus was isolated from only $3.3 \%$ of the blood samples which is in correlation with the reports of Rohani et al., (2000) and Velvizhi et al., (2011).

In our study, we have observed complete resistance $(100 \%)$ to ampicillin and cephalexin. Similarly, Rohani et al., (2000) and Shanthi and Sekar (2009) have encountered $94 \%$ resistance to ampicillin and $82.5 \%$ resistance to ampicillin and cephalexin respectively. 
Fig.1 Distribution of $S$. aureus among various clinical samples

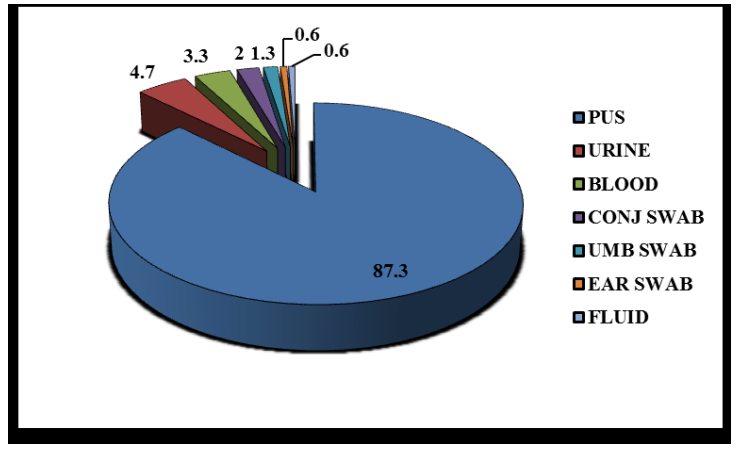

Fig.2 Antibiotic sensitivity pattern of $S$. aureus

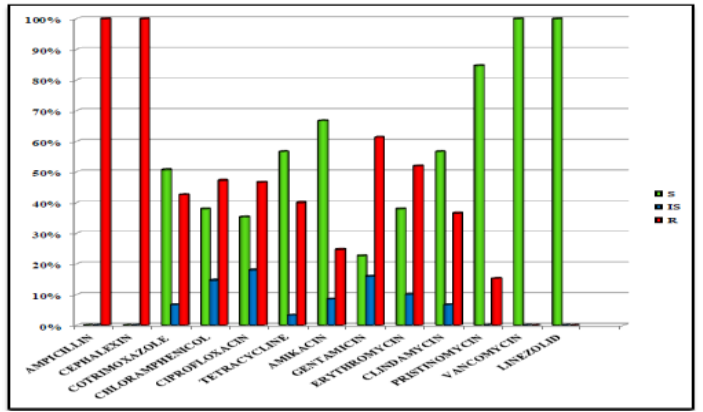

Fig.3 Percentage of MRSA and MSSA among the $S$. aureus

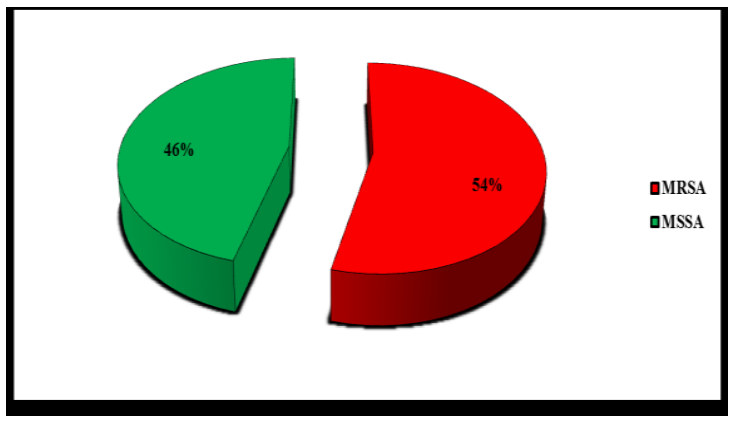

Fig.4 Association of MRSA and MSSA with number of days of hospitalisation

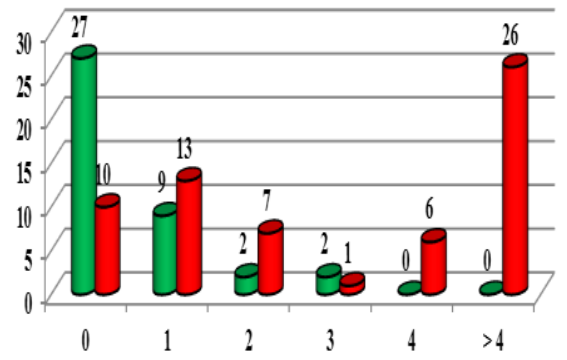


Fig.5 Antibiotic resistance pattern of MRSA and MSSA

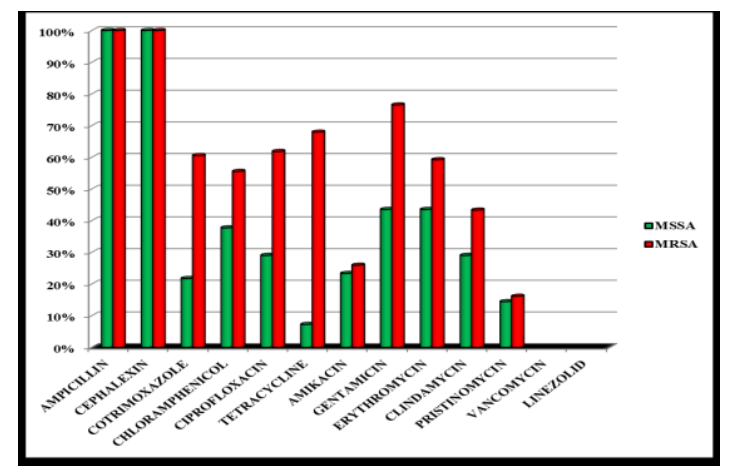

Fig.6 Association of $S$. aureus with prior antibiotic usage

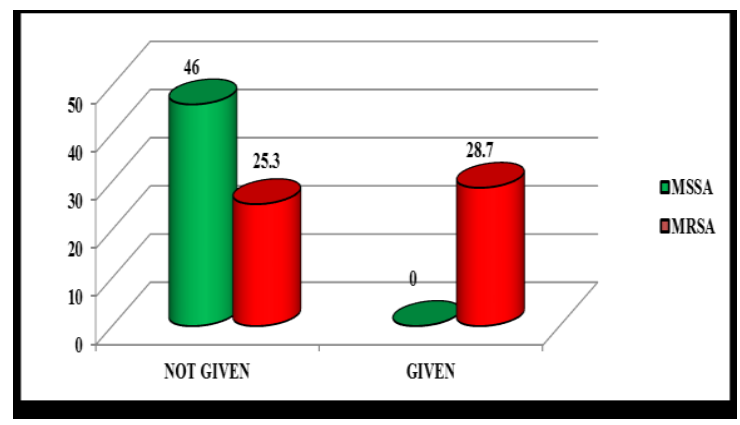

The resistance pattern to various other drugs varied from $15.3 \%$ to $61.3 \%$ which is similar to that of the other studies (Shanthi and Sekar, 2009; Verma et al., 2000; Vardhan et al., 2000; Naik and Deshpande, 2011; Dhanalakshmi et al., 2012.) IT was observed that a total susceptibility of $S$. aureus to vancomycin and linezolid which is in concurrence with other studies (Joshi et al., 2013). The slight variation in the resistance pattern of $S$. aureus to various antibiotics can be attributed to regional variation in the prescription pattern of antibiotics.

Because of the widespread use of antibiotics, especially in developing countries, the resistance profile of microorganisms is changing, evidenced by increasing occurrences of antibiotic resistance among bacterial populations. Additionally, resistance rates are typically higher in developing countries (with rates up to $99 \%$ ) as compared to developed countries (where rates are less than 20\%). Consequently, it is imperative that local surveillance of common pathogenic organisms and their antibiograms be implemented to advise the current use of antibiotics. This is essential to the formulation of prescribing policies based on local statistics.

Our study provides important data on current antimicrobial resistance, including methicillin resistance, for a collection of recent clinical isolates of $S$. aureus from various clinical samples in our hospital. MRSA is a major nosocomial pathogen causing significant morbidity and mortality. There appears to be a significant variable in the epidemiology and prevalence of MRSA in different parts of the world and even in different regions of a country. MRSA since their occurrence in 1961, the incidence has been increasing and a lot of variation in occurrence of MRSA in 
various parts of the country and abroad. As it is causing lots of infections among hospitalised patients and community, MRSA has been targeted for intensive study by various authors.

Now coming to MRSA studies, studies from 1988 till date, range is varying from $6.9 \%$ to $57 \%$. Our study has also shown $54 \%$ of MRSA among S. aureus. Few studies have shown exceptionally high levels of MRSA like Naseer and Jayaraj (2010), Gulbarga, whereas all others are around 50\% for around 8-10 years. Our MRSA pattern is around 54\% which is in comparison with most of the studies done during recent years. Hence, constant monitoring of these strains is essential in order to control their spread in the hospital environment and transmission to the community. In this context, we have also observed about $50 \%$ of CoNS were methicillin-resistant.

All the pathogenic staphylococcal isolates are subjected to MRSA testing routinely. Such a high prevalence of MRSA in our study could be due to several factors. The indiscriminate use of antibiotics, lack of awareness and unethical treatment before coming to the hospital might have been the contributing factors.

There is a lot of variation in the MRSA resistance pattern to the common used antibiotics. In our study, though 100\% resistance was observed to ampicillin and cephalexin, for all the other antibiotics, the resistance pattern was within a limited zone. All these type of variable results need to be kept in mind before administration of any antibiotics in any of the hospital set ups or out-patient clinics. The above graph also indicated that a uniform antibiotic policy has not been followed strictly in India. Therefore, there is a need for formulation of a strict antibiotic policy towards these "notorious bugs" which are taking a lot of time, money and loss of working hours/ labour in the infected patients.

So formulation of a uniform antibiotic policy is the need of the hour. Also, a uniform quality control assessment program must be followed for accurate judgement of susceptibility testing techniques.

It is hoped that the results of this study may be useful to the clinicians in the management of hospitalised patients and out-patients with appropriate antibiotic advice. We expect such studies will also be helpful to the community at large.

Any amount of work on $S$. aureus is not sufficient to demonstrate their virulence factors, which includes resistance to various antibiotics. Maybe, repeated work has to be done in various regions for formulating antibiotic policy.

\section{References}

Anand KB, Agrawal P, Kumar S, Kapila K. Comparison of cefoxitin disc diffusion test, oxacillin screen agar, and PCR for mecA gene for detection of MRSA. Indian J Med Microbiol 2009; 27(1):279.

Anupurba S, Sen MR, Nalti G, Sharma BM, Gulati AK, Moliapati TM. Prevalence of Methicillin resistant Staphylococcus aureus in tertiary referral hospital in eastern Uttar Pradesh. Indian J Med Microbiol 2003; 21(1):49-51.

Arora S, Devi P, Arora U, Devi B. Prevalence of methicillin-resistant Staphylococcus aureus (MRSA) in a tertiary care hospital in Northern India. J Lab Physicians 2010; 2(2):78-81.

Assadullah S, Kakru DK, Thoker MA, Bhat FA, Hussai N, Shah A. Emergence of low-level vancomycin resistance in 
MRSA. Indian J Med Microbiol 2003; 21(3):196-8.

Brown PD, and Ngeno C. Antimicrobial resistance in clinical isolates of Staphylococcus aureus from hospital and community sources in southern Jamaica. Int J Infect Dis, 11(3), 220-5.

Deresinski S. Methicillin-resistant Staphylococcus aureus: an Evolutionary, epidemiologic and therapeutic odyssey. Clin Infect Dis 2005; 40(4):562-73.

Dhanalakshmi TA, Umapathy BL, Mohan DR. Prevalence of inducible clindamycin resistance in Staphylococcus aureus. J Acad Med Sci 2012; 2(2):73-5.

Han Z, Lautenbach E, Fishman N, Nachamkin I. Evaluation of mannitol salt agar, CHROMagar Staph aureus and CHROMagar MRSA for detection of meticillin-resistant Staphylococcus aureus from nasal swab specimens. J Med Microbiol 2007; 56(1):43-6.

Joshi S, Ray P, Manchanda V, Bajaj J, Chitnis DS, Gautam V, et al., Methicillin resistant Staphylococcus aureus (MRSA) in India: Prevalence \& susceptibility pattern. Indian J Med Res 2013; 137(2):363-9.

Lowy FD. Chapter 135. Staphylococcal infections. In: Longo DL, Fauci AS, Kasper DL, Hauser SL, Jameson JL, Loscalzo J, editors. Harrison ${ }^{\text {ee }} \mathrm{s}$ principles of internal medicine. 18th ed. New York: The McGraw-Hill Companies; 2012. vol 1 p. 1160-70.

Moreillon P, Que YA, Glauser MP. Chapter 192. Staphylococcus aureus (including Staphylococcal toxic shock). In: Mandell GL, Bennett JC, Dolin R, editors. Mandell, Douglas and Bennett's: Principles and practice of infectious diseases. 6th ed. Philadelphia: Churchill Livingstone; 2005. vol 2 p. 2321-51.
Naik G, and Deshpande SR. A study on surgical site infections caused by Staphylococcus aureus, with a special search for methicillin-resistant isolates. J Clin Diagn Res 2011; 5(3):502-8.

Naseer BS, and Jayaraj YM. Identification of multi-resistant Staphylococcus aureus in clinical specimens. Research Journal of Medical Sciences 2010; 4(30): 204-7.

National nosocomial infections surveillance report, 2004. Am J Infect Control. 2004; 32(8):470-85.

Rahbar M, and Hajia M. Inducible clindamycin resistance in Staphylococcus aureus: A crosssectional report. Pak J Biol Sci 2007; 10(1):189-92.

Rajaduraipandi K, Mani KR, Panneerselvam K, Mani M, Bhaskar M, Manikandan P. Prevalence and antimicrobial susceptibility pattern of methicillinresistant Staphylococcus aureus: A multicentre study. Indian $\mathrm{J}$ Med Microbiol 2006; 24(1):34-8.

Rohani MY, Raudzah A, Lau MG, Zaidatul AAR, Salbiah MN, Keah KC, et al., Susceptibility pattern of Staphylococcus aureus isolated in Malaysian hospitals. Int J Antimicrob Agents 2000; 13(3):209-13.

Saikia L, Nath R Choudhary B, Sarkar M. Prevalence and antimicrobial susceptibility pattern of methicillinresistant Staphylococcus aureus in Assam. Indian J Crit Care Med 2009; 13(3):156-8.

Shanthi M, and Sekar U. Antimicrobial susceptibility pattern of Staphylococcus aureus at Sri Ramachandra Medical Centre. Sri Ramachandra Journal of Medicine 2009; 2(2):1-4.

Skov R, Smyth R, Larsen AR, Bolmstrom A, Karlsson A, Mills K, et al., Phenotypic detection of methicillin resistance in Staphylococcus aureus by disk diffusion testing and Etest on Mueller-Hinton 
agar. J Clin Microbiol 2006; Vardhan MS, Agarwal DS, Hunter PR. 44(12):4395-9. Methicillin-resistant Staphylococcus aureus in a Delhi teaching hospital. J Hosp Infect. 2000; 46(2):158-9.

resistant Staphylococcus aureus: Prevalence and current susceptibility pattern in Sikkim. J Global Infect Dis 2011; 3(1):9-13.

Udo EE, Al- Sweih N, Noronha B. Characterisation of non- multiresistant methicillin- resistant Staphylococcus aureus (including EMRSA-15) in Kuwait Hospitals. Clin Microbiol Infect 2006; 12(3):262-9.

Van Belkum A, Melles DC, Nouwen J, van Leeuwen WB, van Wamel W, Vos MC et al., Co-evolutionary aspects of human colonisation and infection by Staphylococcus aureus. Infect Genet Evol 2009; 9(1):32-47.

Velvizhi G, Sucilathngam G, Palaniappan N. Prevalence and phenotypic detection of erythromycin-induced resistance to clindamycin in MRSA isolates. J Clin Diagn Res 2011; 5(6):1195-8.

Verma S, Joshi S, Chitnis V, Hemwani N, Chitnis D. Growing problems of methicillin-resistant staphylococciIndian scenario. Indian J Med Sci 2000; 54(12):535-40.

Voss A, Milatovic D, Wallrauch-Schwarz C, Rosdahl VT, Braveny I. Methicillinresistant Staphylococcus aureus in Europe. Eur J Clin Microbiol Infect Dis 1994; 13(1):50-5.

\section{How to cite this article:}

Samira A. W., Samith Ahmed and Meera D. Meundi. 2018. Staphylococcus aureus - A Versatile Pathogen Biochemical Characterization and Antibiogram. Int.J.Curr.Microbiol.App.Sci. 7(02): 2933-2941. doi: https://doi.org/10.20546/ijcmas.2018.702.356 